\title{
A Rare Case of Unusual Urogenital Tract Foreign Body (Plastic Pen) Immigrated from Vagina Due to Masturbation / Autoerotism
}

\author{
Jyotsana Abhay Gawri'1, Manjusha Agrawal² \\ 1,2 Department of Obstetrics \& Gynaecology, Jawaharlal Nehru Medical College, \\ DMIMS, Sawangi, Wardha, Maharashtra, India.
}

\section{INTRODUCTION}

Cases of foreign bodies are seldom seen within the urinary bladder. According to literature, foreign bodies have been excluded from the urinary bladder. Iatrogenic factors, self-infliction or immigration from neighbouring organs can be the cause in such cases. We present an unusual case of 43-year-old middle- aged female who came to our hospital during emergency with complaints of pain abdomen, pain over urethral orificial region \& haematuria caused by a foreign body that was self-inflicted for the purpose of masturbation \& was managed. History in such cases is often deceptive.

Intravesical or intraurethral foreign objects are typically caused by idiopathic injuries, individual's self-insertion, sexual violence, invasion \& immigration from surrounding areas, while immigration from adjacent sites is unusual. ${ }^{1}$

Because of the short urethra \& the closeness to the genitals, the female urinary bladder is fairly easily invaded by foreign objects, both by urethral ascent \& immigration. ${ }^{2}$ The potential driving forces in young women for the introduction of foreign body within the urinary bladder are sexual arousal in young women. Psychiatric illnesses, alcoholism, dementia are significant factors for the incorporation of a foreign object into the urogenital tract. ${ }^{3}$ In the urinary bladder, a variety of different foreign bodies have been identified like electric wires, thermometers, bullets, Intrauterine Contraceptive Devices (IUCD's), wooden sticks, crusted sutures, pencils, domestic batteries, ball point pens, needles, screws, gauze, broken parts of foley's catheters, plastic toys, infant feeding tube, broken endoscopic instrument pieces. In several cases, presentation can be delayed because they fear awkwardness. ${ }^{1}$

Such patients can stay symptomless or have moderate uneasiness but typically manifests with infections of the urinary tract, extreme pain \& haematuria. ${ }^{4}$ Management requires retrieval of foreign artefacts \& external objects must be fully extracted \& the methods used maybe clear \& minimally stressful for the urogenital tract. ${ }^{4}$ Herein, we present case \& management of self-inflicted foreign body in urethra in a middle-aged woman for erotic stimulation.

\section{PRESENTATION OF CASE}

A 43-year-old middle-aged widow presented to our emergency department with the complaints of lower abdominal pain, pain over urethral region, dysuria \& haematuria. On proper history taking, initially denied actual history \& then patient reported insertion of plastic pen through the urethra for the purpose of masturbation. The pen accidentally slipped into the bladder \& patient was referred to emergency room one day after the incident because of symptoms. She reported normal menstrual history. There was no associated psychiatric illness. Routine laboratory investigations showed normal blood count \& electrolyte profile, but urine analysis showed plenty of pus cells \& microscopic RBC's in urine. Henceforth, a urine culture was sent \& injection ceftriaxone $1 \mathrm{gm}$ intravenously twice daily was started empirically. On per abdomen examination some longitudinal tubular structure was palpable below the umbilicus.

A plain radiograph KUB (Kidney Urinary Bladder) was performed which showed a foreign body with a metal density in the pelvic area, one end was pointed \& another end was tubular \& wider. Lying obliquely in the pelvis was seen as low-density circular object which seemed to be associated with the smaller foreign body.
Corresponding Author: Dr. Jyotsana Abhay Gawri, Department of Obstetrics \& Gynaecology, Jawaharlal Nehru Medical College, DMIMS, Sawangi, Wardha, Maharashtra, India. E-mail: sanagawri@gmail.com

DOI: $10.14260 / j e m d s / 2020 / 766$

How to Cite This Article: Gawri JA, Agrawal M. A rare case of unusual urogenital tract foreign body (plastic pen) immigrated from vagina due to masturbation / autoerotism. J Evolution Med Dent Sci 2020;9(46):3500-3502, DOI: 10.14260/jemds/2020/766

Submission 08-08-2020,

Peer Review 02-10-2020,

Acceptance 08-10-2020,

Published 16-11-2020.

Copyright (C) 2020 Jyotsana Abhay Gawri et al. This is an open access article distributed under Creative Commons Attribution License [Attribution 4.0 International (CC BY 4.0)] 
Ultrasonography of kidney, ureter and bladder showed significant post void residual urine with distended bladder \& both kidneys appeared to be normal. A contrast enhanced Computed Tomography (CT) \& CT cystogram was done which revealed evidence of a long tubular oblique structure noted within urinary bladder with length of around $12 \mathrm{~cm}$ with radio-opaque area at one tip with no obvious extravasation of contrast into peri-vesical space suggestive of foreign body. Its posterior end was lateral to right vesico-ureteric junction \& another tip in anterior superior surface with focal peri vesicle fat stranding. The shape of foreign body was resembling a plastic pen. Once a diagnosis of foreign body in bladder was confirmed, cystoscopic removal of foreign object was performed after informed valid consent \& a plastic pen was removed without any surgical or post-surgical complications.

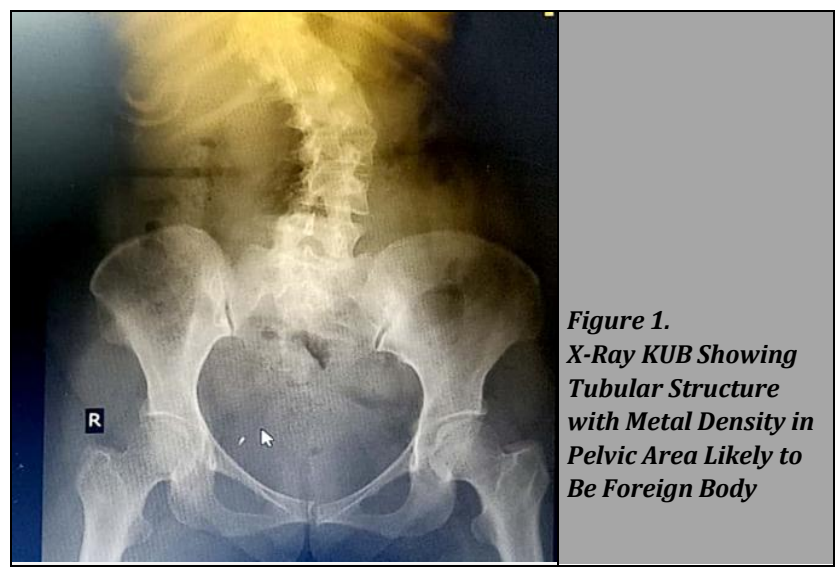

DISCUSSION

Self-insertion of foreign objects into the urethra or bladder more commonly occurs as a result of attempted sexual stimulation, intoxication or psychiatric disease, ${ }^{5}$ migration from adjacent organs, via urethra or traumatic route. Different foreign intravesical objects documented are surgical gauze, intrauterine devices, ball point pens, lead pencils, domestic batteries, broken endoscopic tool parts, metallic wires, broken parts of foley's balloon catheter parts, needle etc. ${ }^{4}$ Foreign objects in the urogenital tract is not unusual though records are not available regarding the exact frequency of occurrence in India or around the world. In Medline quest only single case records or series of cases are available. Rafique $\mathrm{M}$ in Urol J reported 17 cases of intravesical foreign bodies in a span of five years. ${ }^{6}$

The key mechanism in other studies was the ascending migration or self-insertion of a foreign object through the urethra into the bladder. In a study done by Wachira Kochakarn et al, it was found that this process to be the main route for the entry of foreign bodies into the bladder. Females are more vulnerable to ascending migration of foreign bodies than males because of shorter urethra \& its straight alignment. Because the urethra's position is not clearly visible, a foreign object can mistakenly be inserted while masturbation. ${ }^{2}$

In most of the cases, clinical sensitivity varies from asymptomatic to external genital swelling, pain abdomen, haematuria, dysuria, reduced urinary flux or retention, ascending urinary tract infections. ${ }^{7}$ Rose Cury et al reported retroperitoneal migration of ball point pen on self-insertion via the urethra, a case similar to our case. ${ }^{8}$
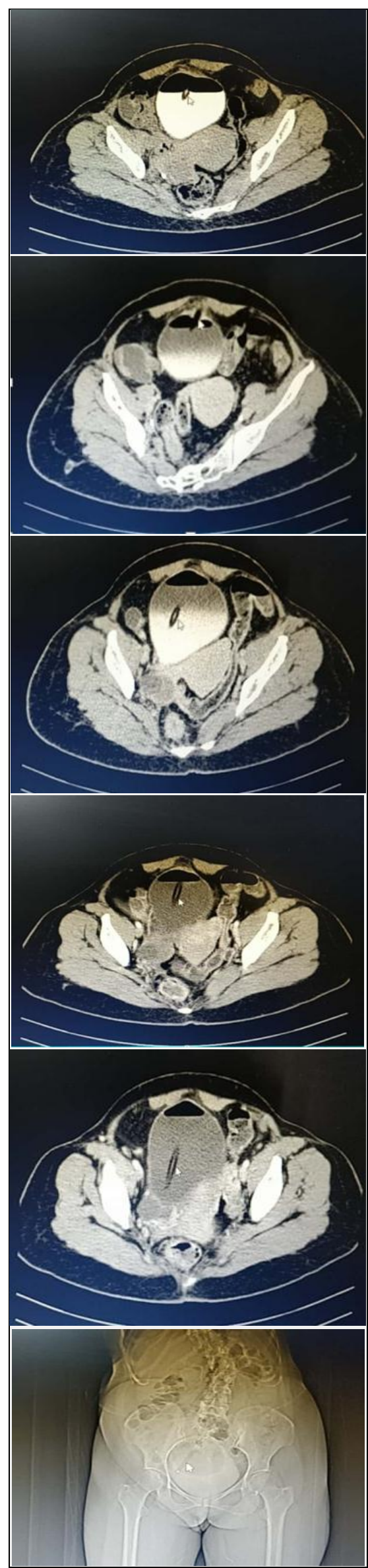

Figure 2

CECT Abdomen, Pelvis Shows Evidence of Hypodensity with Inner Hyperintensity Noted in Urinary Bladder. On Delayed Scan, It is Appearing as Filling Defect 
In our case, the configuration of foreign object led to bladder mucosal \& urethral injury with signs and symptoms of pain abdomen $\&$ haematuria.

In terms of management \& based on nature of foreign body, its location, shape \& size, different methods of removal has been described. Radiological evaluation helps to determine the actual size, position \& number of foreign object. ${ }^{4}$ Evidence can be accurately obtained for radio-opaque foreign materials with a simple kidney urinary bladder radiograph, ultrasound, computed tomography \& radiolucent foreign bodies can be identified with the help of ultrasonography \& MRI (Magnetic Resonance Imaging). ${ }^{6}$ However, the most effective tool for diagnosing intravesical foreign bodies remains urethrocystoscopy. These days' endoscopic procedures are the preferential treatment modalities because they reduce injuries of lower urinary tracts. In few cases however, to minimize the risk of urethral \& bladder injury open procedures like suprapubic cystostomy is still recommended. Since female bladder can be assessed easily via urethra, foreign objects can be safely removed endoscopically. Routine psychiatric evaluation is recommended as high incidence psychiatric disease, mental retardation is reported in these patients. ${ }^{4}$ Urogenital fistula may be a result of penetration of foreign bodies into urogenital tract. Urogenital fistula management depends on the size of the defect \& its location.

In the present situation, the foreign body was advanced to bladder from vaginal site through a perforation of bladder. Through clinical history, pelvic radiograph, sonography \& CT scan a diagnosis of foreign body was established. The foreign body was successfully removed endoscopically through cystoscopic guided approach. This case supports the view that endoscopic methods should be the treatment of first choice in extracting foreign materials from the urinary bladder \& urethra. Correct diagnosis \& history taking are important steps when a foreign object is suspected.

\section{CONCLUSIONS}

Foreign object may be seen in the urinary bladder with various symptoms, signs \& the history is often deceptive. When patient complains of lower abdominal pain, dysuria, fever, other urinary symptoms a suspicion of foreign body should be kept in mind especially in psychiatric patients. A foreign body can be detected by detailed history \& clinical examination. However, imaging modalities like pelvic radiograph, ultrasonography, computed tomography scan \& endoscopy (cystoscopy) maybe required. With advances in endoscopic procedures, most cases can be successfully treated using minimally invasive techniques. Prolonged catheterization helps in healing of small vesicovaginal fistulas spontaneously.

Financial or other competing interests: None.

Disclosure forms provided by the authors are available with the full text of this article at jemds.com.

\section{REFERENCES}

[1] Bansal A, Yadav P, Kumar M, et al. Foreign bodies in the urinary bladder and their management: a single-centre experience from North India. Int Neurourol J 2016;20(3):260-9.

[2] Kochakarn W, Pummanagura W. Foreign bodies in the female urinary bladder: 20-year experience in Ramathibodi Hospital. Asian J Surg 2008;31(3):130-3.

[3] Ortoğlu F, Gürlen G, Altunkol A, et al. A Rare foreign material in the bladder: piece of pencil. Arch Iran Med 2015;18(9):616-7.

[4] Bansal A, Kumar M, Kanodia G, et al. Unusual intravesical foreign body in young female migrated from vagina due to autoerotism. Int Braz J Urol 2017;43(3):556-60.

[5] MacCraith E, Davis NF, Forde J. Unusual genitourinary tract activity. BMJ Case Rep 2017;2017:bcr2017220297.

[6] Rai GS, Roshan R, Sarawagi R, et al. Acute pelvic pain: a ball pen may be a cause? J Clin Diagn Res 2014;8(12):RD04-5.

[7] Bantis A, Sountoulides P, Kalaitzis C, et al. Perforation of the urinary bladder caused by transurethral insertion of a pencil for the purpose of masturbation in a 29-year-old female. Case Rep Med 2010;2010:460385.

[8] Cury J, Coelho RF, Srougi M. Retroperitoneal migration of a self-inflicted ballpoint pen via the urethra. Int Braz J Urol 2006;32(2):193-5. 